\title{
Excepciones a derechos Exceptions to rights
}

\author{
Marcos Andrade Moreno \\ Universidad Católica Silva Henríquez \\ mandrade@ucsh.cl \\ Universitat Pompeu Fabra \\ marcos.andrade@upf.edu
}

Recibido / received: 20/02/2018

Aceptado / accepted: 06/03/2018

DOI: https://doi.org/10.20318/eunomia.2018.4166

\section{Resumen}

El artículo presenta dos sentidos de excepciones a derechos y las razones por las que conviene distinguirlos. Se muestran las dificultades teóricas de definir el concepto de excepción y cancelación o suspensión de derechos, ya que ellos, al parecer, descansan en la idea de anormalidad. Se muestran las dificultades de este enfoque y su justificación en el debate en torno a las excepciones implícitas y la derrotabilidad. Se ofrece una manera de superar estas dificultades, abandonando el enfoque lógico. En su lugar, se presenta un enfoque funcional que busca co-definir las excepciones y las suspensiones o cancelaciones mediante sus relaciones con otras normas denominadas normas alterativas, cuyo fin sería alcanzar cierto gran de flexibilidad normativa.

\section{Palabras clave}

Excepciones, cancelaciones, normas que establecen derechos, flexibilidad normativa.

\begin{abstract}
The paper presents two meanings of exceptions to rights and why it is convenient to distinguish them. The theoretical difficulties of defining the concept of exception and derogation of rights based on the idea of abnormality are shown. Instead, by showing the difficulties of this approach and its justification on the implicit exceptions and defeasibility debate, it offers a way to overcome these difficulties abandoning the logical approach. In lieu, a functional approach is presented: one that seeks to co-define exceptions and suspensions or cancellations through their relationships with other norms called alterative norms, whose purpose would be to achieve a certain degree of normative flexibility.
\end{abstract}

\section{Keywords}

Exceptions, derogations, enacted rights, normative flexibility.

SUMARIO. 1. Sentidos de la excepción. 2. Excepciones. 3. Excepciones y suspensión de derechos. 4. Conclusión. 
También esto es de Focílides:

los lerios son unos malvados, no unos sí y otros no, sino todos excepto Procles;

y Procles es un lerio! ${ }^{1}$

\section{Sentidos de la excepción}

En lo sucesivo se presentarán dos sentidos en que puede emplearse la expresión 'excepción a un derecho', se mostrará por qué conviene distinguirlos y cuál es la importancia de dicha distinción para la comprensión de las normas que establecen derechos.

En un sentido, 'excepción' puede hacer referencia a una cláusula establecida prima facie en la formulación de un derecho, que sustrae a priori, de manera permanente y con efectos generales, del alcance o de la extensión de la protección de un derecho, un determinado ítem que de otra manera debería entenderse incluido en la norma que establece un derecho.

En el otro sentido, 'excepción' apunta a una cláusula establecida prima facie que cancela o suspende a posteriori la protección de un derecho de manera esencialmente transitoria, introduciendo unos elementos externos genéricos, ciertos estados de cosas, que el instrumento que establece derechos preselecciona y cuyo acaecimiento autoriza a activar dicha suspensión y su control por parte del tribunal.

Estos dos sentidos suelen confundirse. Por ejemplo, se habla en general de excepciones como restricciones a derechos: "cualesquiera medidas, de alcance general o particular, que reducen el ámbito de aplicabilidad de un derecho" (DíezPicazo, 2003, p. 103); razón por la cual, se considera que dichas restricciones incluyen las suspensiones o cancelaciones a derechos, al ser ellas una "variedad de la restricción de derechos fundamentales" (Díez-Picazo, 2003, p. 116).

Esta confusión se debe en gran medida a Schmitt, quien famosamente consideraba que: "La excepción es más interesante que el caso normal. Lo normal nada prueba; la excepción, todo; no sólo confirma la regla, sino que ésta vive de aquélla" (2009, p. 20). ¿Pero en qué consiste esta normalidad? El punto de vista Schmitt descansa sobre un presupuesto de la normatividad del derecho:

\footnotetext{
"Toda norma general requiere que las relaciones vitales a las cuales ha de ser aplicada efectivamente y que han de quedar sometidas a su regulación normativa, tengan configuración normal. La norma exige un medio homogéneo. Esta normalidad fáctica no es un simple "supuesto externo» que el jurista pueda ignorar; antes bien, es parte de su validez inmanente. No existe una sola norma que fuera aplicable a un caos. Es menester que el orden sea restablecido, si el orden jurídico ha de tener sentido" (Schmitt, 2009, p. 18).
}

Esta configuración por defecto de un trasfondo homogéneo, esta normalidad fáctica implícita, permite entender aquello que sería común a excepciones y suspensiones: dado que ellas estarían contempladas prima facie en el sistema

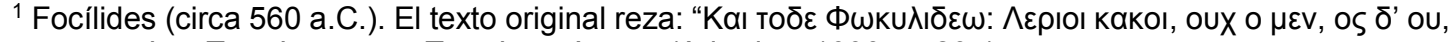

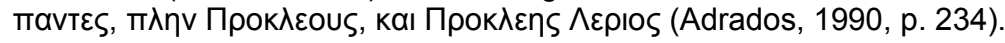


jurídico, existe en principio una justificación de fondo para ellas que, en este caso, sería compartida, a saber, el de anormalidad o excepcionalidad ${ }^{2}$.

No obstante, justamente por ello, Schmitt las confunde: porque ambas compartirían la situación de anormalidad que sería la condición de aplicación explícita de ellas, en la medida de que lo implícito a todas las normas jurídicas sería la configuración normal del orden jurídico ordinario.

Por el contrario, esto es justamente lo que hace diferentes a las excepciones y a las suspensiones, ya que "donde se evocan las excepciones a la regla, Schmitt teoriza sobre la situación excepcional". Por esta razón, si uno "rechaza los postulados teóricos schmittianos, ya no se está obligado a mantener esta fusión de la excepción como anormalidad (como anormalidad, como fuera de lo común, como insubsumible) y excepción como un acto de excepción a una regla" (Carpentier, 2014, p. 8) ${ }^{3}$.

En otras palabras, la guerra, las emergencias graves, las calamidades públicas, entre otras, son situaciones excepcionales, estados de cosas que el propio sistema jurídico contempla, correspondiéndoles cierta consecuencia jurídica: la declaración de estado de guerra, sitio o excepción. Estos son a su vez elementos que la norma que establece derechos contempla y que permiten activar la suspensión de la protección de los derechos, que el tribunal debe controlar.

Por el contrario, en el caso de las excepciones, no existen tales situaciones excepcionales, estados de cosa anormales, que afecten al orden jurídico, sino que, más bien, lo que está en juego es otra cosa: dada la generalidad de las normas, hay ciertos casos cuyo acaecimiento el legislador desea dejar fuera del alcance de la norma anticipadamente, previniendo su futuro incumplimiento 4 .

Así, parece que el problema está del lado de las excepciones, ya que si bien el legislador contempla prima facie qué casos quedan fuera del alcance de la norma de forma explícita, es imposible que contemple todos los casos, aceptándose la posibilidad de que existan excepciones implícitas. En otras palabras, las excepciones son posibles gracias a "la generalidad de las leyes y reglas", pero, por lo mismo, "las reglas jurídicas son objeto de excepciones actuales o potenciales" (Honoré, 1977, p. 108).

Esto se debe a la naturaleza general de las normas jurídicas y a la estructura con que tradicionalmente se concibe a las normas prescriptivas: "la conjunción de dos elementos, el supuesto de hecho y la consecuencia jurídica, unidos ambos por un nexo. De manera que la norma jurídica se representaría de acuerdo al siguiente esquema: "Si es A, debe ser B»"5. Por ello, es posible concebir a las normas jurídicas

\footnotetext{
2 En este sentido: “El autor de la regla 'hace' una excepción, porque la regla, sin ninguna excepción particular, sería insatisfactoria, desde el punto de vista de las justificaciones de fondo de la regla o los poderes normativos fuera de ellas" (Carpentier, 2014, pp. 17-18).

${ }^{3}$ Estas críticas a Schmitt no son nuevas, Mathias Schmitz en los 60 y Julien Freund en los 70 ya las habían identificado. Para una presentación de estas y otras críticas a Schmitt, véase Gómez, 1986, pp. 39-40. Esto redunda en que es posible separar, al menos conceptualmente, la excepcionalidad del estado de excepción (véase Honig, 2009, pp. 66-69).

${ }^{4}$ Para un desarrollo de estas ideas, véase Carpentier, 2014, p. 9.

5 Se dice también que en la estructura de la norma jurídica es posible discernir "entre supuesto de hecho y consecuencia jurídica" (Moreso y Vilajosana, 2004, p. 72). En este sentido, la norma jurídica es entendida como "un imperativo hipotético en el que la aplicación de la consecuencia jurídica es el resultado del efectivo acaecimiento del caso previsto en el supuesto de hecho" (Ansuátegui, 1999, p. 153). Por esta razón, para Kelsen la norma funciona como un esquema de explicitación: "El acontecimiento en cuestión logra su sentido específicamente jurídico, su significación propia en derecho, a través de una norma que se refiere a él con su contenido, que le otorga significación en derecho, de suerte que el acto puede ser explicitado según esa norma" (1986, p. 17). Es justamente a esta
} 
como universales derrotables ${ }^{6}$. Aquí es justamente donde reside el problema, porque como señaló Hart:

"Por lo general, no es posible definir un concepto jurídico como 'traspaso' o 'contrato' especificando las condiciones necesarias y suficientes para su aplicación. Para cualquier conjunto de condiciones puede ser adecuado en algunos casos, pero no en otros, y tales conceptos solo se pueden explicar con la ayuda de una lista de excepciones o ejemplos negativos que muestran que el concepto no se puede aplicar o solo se puede aplicar de forma debilitada [...] Esta característica de los conceptos jurídicos es una para la cual no existe una palabra en inglés común. Las palabras 'condicional' y 'negativo' tienen las implicaciones incorrectas, pero el derecho tiene una palabra que con cierta vacilación tomo en cuenta y extiendo: esto es el sentido 'derrotable' usado de un interés legal en la propiedad que está sujeta a terminación o 'derrota' en una cantidad de contingencias diferentes, pero permanece intacto si tales contingencias no maduran" (1948-1949, p. 174)

Así, las normas (N1) 'Cualquiera que mate a otro ser humano es culpable de homicidio' y (N2) 'Cualquiera que mate a otro ser humano en defensa propia no es culpable de homicidio', pueden ser ambas válidas en un sistema jurídico. ¿Cómo es posible explicar esto? ${ }^{8}$ La explicación es que, como se ha dicho "Formulamos nuestras afirmaciones para circunstancias normales, sabiendo que en ciertas situaciones nuestros enunciados serán derrotados", por ello "[m]uchas (si no todas) las formulaciones normativas son derrotables, esto es, poseen usualmente excepciones implícitas, existen circunstancias que derrotan la norma, aunque ellas no estén explícitamente enunciadas" (Alchurrón, 1991, p. 24).

Esto nos vuelve al problema planteado por Schmitt, ya que justamente la diferenciación entre excepciones y suspensiones o cancelaciones, que analizamos

concepción de las normas jurídicas a la que se opone Schmitt: "Afirmar que lo excepcional no tiene importancia jurídica y que es propio de la «sociología», sería tanto como dar por buena la esquemática disyunción de la sociología y la teoría del derecho. Lo excepcional es lo que no se puede subsumir; escapa a toda determinación general, pero, al mismo tiempo, pone al descubierto en toda su pureza un elemento específicamente jurídico, la decisión". Por ello, se "comprende que un neokantiano como Kelsen no acierte a dar cabida en su sistema al estado excepcional” (2009, pp. 18-19). También, véase Schmitt, 1996.

${ }^{6}$ Esto quiere decir que "en el lenguaje corriente, las construcciones condicionales de la forma 'Si A entonces B' son frecuentemente usadas de modo tal que no se pretende con ellas afirmar que el antecedente $A$ es condición suficiente del consecuente $B$, sino sólo que el antecedente, sumado a un conjunto de presupuestos aceptados en el contexto de emisión condicional, es condición suficiente del consecuente B [...] La afirmación condicional es derrotada cuando alguno de los presupuestos implícitos resulta falso. Un condicional derrotable puede ser definido como un condicional sujeto a excepciones implícitas" (Alchurrón, 2000, p. 23). Por ello, se dice que muchos teóricos del derecho "encuentran plausible pensar las reglas jurídicas como condicionales universales que correlacionan consecuencias normativas al acaecimiento de ciertos hechos o hechos. Parece también plausible pensar que las reglas jurídicas pueden ser sujetas a excepciones. Pero es normal considerar que estas dos ideas son incompatibles, y que ambas no pueden ser acomodadas cuando intentamos dar cuenta de la forma de las reglas jurídicas" (Duarte D’Almeida, 2015b, p. 133).

${ }^{7}$ También en otra parte señala que: "Podríamos hacer reglas, cuya aplicación a casos particulares nunca requiera una opción adicional. Cada cosa se podría conocer, y para todo, ya que se podría saber, hacerse y especificarse de antemano por la regla. Este sería un mundo apto para la jurisprudencia 'mecánica'. Es evidente que este mundo no es nuestro mundo, los legisladores humanos no pueden tener tal conocimiento de todas las posibles combinaciones de circunstancias que el futuro puede traer [...] Cuando un caso no previsto surge, enfrentamos la cuestión escogiendo entre los intereses en competencia de la manera que mejor nos satisface. Al hacerlo, habremos resuelto de modo más determinante nuestro objetivo inicial e, incidentalmente, habremos resuelto una cuestión sobre el significado, para los propósitos de esta regla, de una palabra general" (Hart, 1997, pp. 128-129).

8 Como dice Alchurrón: "En relación con un condicional derrotable, puede ser verdadero que 'Si A entonces B' y falso que 'Si A y C entonces B'. En tal caso, la circunstancia C constituye una excepción que derrota el condicional 'Si A entonces B' [...] Es decir, un condicional es derrotable si el siguiente esquema de inferencia (denominado refuerzo del antecedente) es lógicamente inválido: de 'Si $\mathrm{A}$ entonces B' se sigue que 'Si A y C entonces B'” (Alchurrón, 1991, pp. 23-24). 
antes, parte del supuesto de que se puede distinguir entre la situación excepcional que comportan estas últimas y la situación de normalidad que comportan las primeras. Esto es así debido a que, si siempre podemos encontrar circunstancias que derrotan la norma no contempladas en ella, por más precisa y rigurosamente que las definamos en las cláusulas exceptiva o suspensiva, no sería posible distinguir entre ambas, porque el trasfondo homogéneo o la normalidad fáctica implícita no sería tal.

En este artículo pretendo mostrar por qué, a pesar de este problema, es posible distinguir ambos sentidos de excepción, ya que lo relevante estaría no en dicha justificación de trasfondo homogéneo o normalidad fáctica implícita, sino que en los poderes normativos que las normas que establecen derechos tienen fuera de ellas.

Como se verá, se debe mostrar en primer lugar cómo se sostiene la distinción, es decir, cómo podemos identificar cuándo estamos frente a una excepción y cuando frente a una cancelación. Ello supone abandonar cualquier intento de identificación mediante determinados marcadores semánticos presentes en la norma que establece derechos. En su lugar se debe adoptar un criterio funcional, el que supone una reconstrucción sistemática de las excepciones y las cancelaciones como pertenecientes a una categoría más amplia de normas: las normas alterativas.

De esta manera, se puede comprender de qué manera las excepciones y cancelaciones alteran o modifican los poderes normativos de las normas que establecen derechos y, de paso, por qué ellas permiten alcanzar un cierto grado de flexibilidad normativa, indispensable en la determinación de la protección de los derechos.

\section{Excepciones}

¿Cómo es posible entonces que las normas (N1) 'Cualquiera que mate a otro ser humano es culpable de homicidio' y (N2) 'Cualquiera que mate a otro ser humano en defensa propia no es culpable de homicidio' sean ambas válidas en un sistema jurídico? Hay dos estrategias que se han propuesto sobre este asunto.

La primera, llamada internalista o incorporacionista, consiste en "admitir que una descripción rigurosa de la primera regla debería incorporar una cláusula que estableciera que el acto de matar no es cometido en defensa propia" (Duarte D'Almeida, 2015b, p. 135) ${ }^{9}$. En un pasaje célebre de Taking Rights Seriously, Dworkin señala:

"En el béisbol, una regla establece que si el batter no contesta tres lanzamientos, queda fuera del juego. No es coherente reconocer que ésta es una enunciación correcta de las reglas del béisbol y decir que un bateador que no contesta tres lanzamientos no queda fuera del juego. Por cierto, que una regla puede tener excepciones [...] Sin embargo, un enunciado preciso de la regla tendría en cuenta en cuenta esta excepción, y cualquier enunciado que no lo hiciera sería incompleto. Si la lista de excepciones es muy grande, sería demasiado incómodo repetirlas cada vez que se cita la regla; en teoría, sin embargo, no hay razón por la cual no se las pueda agregar a todas; y, cuantas más haya, tanto más preciso es el enunciado de la regla" (Dworkin, 2012: 75).

Irónicamente, quizás el otro famoso defensor del ideal de que es posible listar todas las excepciones a las normas sea Bentham ${ }^{10}$. En cualquier caso, como

\footnotetext{
${ }^{9}$ Contra esta tesis estructura su libro sobre el tema, véase Duarte D'Almeida, 2015a.

10 Sobre este ideal de Bentham se ha dicho que: "El amor de Bentham por la luminosa claridad y certeza de la ley, lo llevó al sueño de una reconstrucción ideal de las reglas legales que contuviera todas las
} 
Finkelstein remarca, la posición interpretativista sobre las excepciones tiene como consecuencia que la regla se trague la excepción, porque ellas serían una parte implícita de la regla: no hay calificaciones fuera de la regla. Así, el punto de vista internalista sobre las excepciones "sugiere que todas las excepciones a una regla pueden determinarse interpretando la regla correctamente [...] La noción de excepción a una regla queda excluida por la comprensión de las reglas que sugiere Dworkin" (Finkelstein, 2000, p. 509) ${ }^{11}$. En este mismo sentido se pronuncia Atria, cuando afirma que:

“Hablar de 'excepciones' podría ser engañoso en un sentido importante: las excepciones son cláusulas que limitan el alcance de una regla, por lo tanto, por definición, si hay una excepción, la regla no es aplicable. Por lo tanto, parece que las excepciones son imposibles según el criterio de Miller: para que exista una excepción a una regla, la regla debe ser aplicable, pero si existe una excepción, la regla no es aplicable." (2001, p. 124).

En otras palabras, las reglas tienen que ser aplicables para que estemos en presencia de una excepción que la desaplica ${ }^{12}$.

La segunda, Ilamada externalista o no-deductiva, señala que "si queremos seguir sosteniendo que la primera regla debe ser formulada, como originalmente lo fue, sin mencionar la defensa propia - al fin y al cabo, pensamos en la defensa propia como una excepción a esa regla-, tenemos que admitir que nuestras reglasenunciados no especifican condiciones suficientes para las consecuencias correspondientes" (Duarte D’Almeida, 2015b, p. 135).

La posición externalista sostiene que las excepciones son externas a la norma que se aplica, no están implícitas en ellas. Por ello, Schauer sostiene que: "Solo cuando la designación primaria del ámbito de aplicación de una norma incluye, en vez de excluir, un cierto ítem que sus creadores desean no incluir, es necesario añadir una excepción" (Schauer, 1991, p. 874) $)^{13}$.

En el caso de Schauer, su visión externalista de las excepciones termina confundiendo las excepciones con un caso de derrotabilidad, ya que en las

excepciones posibles. Ahora tenemos buenas razones para ser un poco más escépticos" (Moreso, 2014, p. 9).

${ }^{11}$ Esta posición de Dworkin es una reacción a la idea de Hart de que es imposible hacerlo, con lo que inaugura el tema de la derrotabilidad de las normas jurídicas. Sin embargo, el problema de las excepciones es un problema de aplicabilidad, no de pertenencia de las normas. Dado que Dworkin no maneja la distinción entre pertenencia y aplicabilidad de una norma (véase Moreso y Navarro, 1996), tiene que ser capaz de contestar la siguiente objeción: mediante una excepción nos encontraríamos en el caso de que una regla sea válida y se pueda aplicar al supuesto, al mismo tiempo de que ella misma establece que no se aplica. Si no fuere así, la regla estaría incompleta. Por eso Dworkin fracasa en su caracterización de las excepciones dentro de un sistema jurídico, dado que "no hay tal cosa como una excepción a una regla, ya que cualquier excepción articulada es solo una declaración más precisa de la regla" (Finkelstein, 2000, p. 510). Por ello, la excepción desaparece engullida por la regla. Esta consecuencia no se resuelve acudiendo a los principios, ya que éstos no son decisivos en el caso.

12 Como dice Finkelstein: "Es razonable suponer que una condición calificada solo puede constituir una excepción si la cosa que califica es dispositiva del resultado en ausencia de la calificación en un caso al cual se aplica" (2000, p. 510).

${ }^{13}$ Por ello, para Schauer: [L]a necesidad de una excepción (o la falta de ella) a alguna regla legal es en gran medida una función de la gama de herramientas lingüísticas disponibles para el redactor de la regla. Cuando el lenguaje en el que se redacta la regla contiene una palabra o frase familiar que por sí misma excluye lo que los redactores desean excluir del alcance de la regla, no es necesaria ninguna excepción. Todo lo que se requiere es emplear la palabra o frase apropiada, y lo que se debe excluir se excluye sin la necesidad de una excepción. Pero cuando el lenguaje no proporciona ninguna palabra o frase, el alcance de alguna prescripción o proscripción primaria se definirá en términos que probablemente sean excesivamente incluyentes, desde la perspectiva de los objetivos de la regla legal. Para adaptar la regla a los objetivos legales, entonces es necesario crear una excepción (1991, p. 874). 
excepciones explícitas el intérprete reconocería que la aplicación de la regla cambia el trasfondo implícito normal, lo que altera el propósito original de la norma. En otras palabras, permitir una excepción a una regla resulta en "cambiar la regla y crear una nueva regla que incluya la condición calificadora relevante dentro de su enunciación" (Finkelstein, 2000, p. 510), lo que se justifica en la función o propósito original que la regla poseía. Esta aproximación es casuística, de lo contrario "la regla perdería toda la fuerza normativa, ya que solo se aplicaría en aquellos casos en los que el resultado alcanzado aplicando la regla podría alcanzarse directamente desde la justificación de fondo de la regla" (Finkelstein, 2000, pp. 510-511) ${ }^{14}$.

Con razón se ha sostenido que tanto la postura internalista como la externalista son insatisfactorias. La primera, porque se basa en la idea de "encapsular en el antecedente de la regla, la negación de la disyunción de las excepciones admisibles" y, por tanto, "produce reglas con antecedentes abiertos e inservibles". También debido a que "trataría la ausencia de cada excepción admisible meramente como otro elemento en el antecedente de la regla [...] esto es algo que nos resistimos a aceptar, ya que nos parece que sí hay una distinción a trazar entre elementos «negativos» de la regla [...] y excepciones propiamente dichas" (Duarte D'Almeida, 2015b, p. 135).

La segunda, porque "nos compromete con un enfoque no-monotónico de la justificación del derecho", por lo que "implicaría abandonar una idea conocida aparentemente plausible: la idea de que las conclusiones del derecho pueden ser presentadas como conclusiones que se siguen deductivamente de alguna combinación de descripciones de reglas jurídicas y descripciones de los correspondientes hechos" (Duarte D'Almeida, 2015b, p. 135).

En otras palabras: "Un aspecto del problema, es cuáles son los límites de la precisión o exhaustividad en los enunciados jurídicos; el otro aspecto, es cuál es la confiabilidad con la cual uno puede hacer inferencias a partir de un enunciado expreso en que ley se formula" (Maccormick, 1995, p. 103). Por ello, las estrategias fallan respectivamente en dos aspectos relevantes del problema: en la exhaustividad con la que podemos reconstruir y en la confiablidad de nuestras inferencias sobre las normas a partir de los enunciados normativos.

¿Existe alguna alternativa a estas dos posturas? Recientemente se ha planteado la estrategia eso es todo, que busca dar respuesta al argumento de la superación, es decir, la idea de que siempre podría encontrarse una excepción a una norma mostrando una característica adicional no contemplada que muestre la inadecuación de la norma ${ }^{15}$.

Esta estrategia surge desde el particularismo. Como señala Dancy: "Al fin y al cabo un principio moral especifica una consideración como una razón moral. Por ejemplo, el principio según el cual es incorrecto mentir toma un rasgo, la mendacidad,

\footnotetext{
${ }^{14}$ Del mismo modo, según "[e]l pensamiento de Schauer parece ser que agregar una excepción en el momento de la aplicación equivale a cambiar una regla, pero que reconocer una excepción articulada previamente no lo es" (Finkelstein, 2000, p. 511). Por ello, Schauer concluye que las reglas deben modificarse solo cuando su aplicación en un caso particular produzca un resultado absurdo.

15 "[D]ado cualquier acción cuyas características se describen en términos no morales, y un principio que dice que una acción que tenga esas características será buena, siempre podemos pensar en alguna característica adicional que sea tal que, si la acción tuviera esa característica también, se convertiría en una acción incorrecta. Entonces el principio es inadecuado tal como está. Debe ser modificado, o se debe dar otro principio que describa la excepción. Pero una vez que hayamos hecho eso, se encontrará una excepción a los principios enmendados y seríamos forzados a enmendarla nuevamente, y así sucesivamente" (Holton, 2002, p. 198).
} 
y dice que cada acción que posea este rasgo es incorrecta" $\left(2015\right.$, p. 20) ${ }^{16}$. Pero justamente encontraremos circunstancias en que una acción que posea este rasgo sea correcta: como mentir para salvar la vida de un inocente, en el famoso ejemplo de Sartre. Por ello, el punto de vista de los particularistas es que

"[l]as reglas son generalizaciones que van desde el pasado hacia el futuro. Pero nada nos garantiza que acierten [...que] haya alguna circunstancia no prevista en las condiciones de aplicación de la formulación de la regla que arroje una solución normativa diversa, e incompatible, de aquella prevista por la regla -i.e. la generalización- [...] Las reglas, por lo tanto, son generalizaciones falibles." (Luque, 2015, p. 12) ${ }^{17}$.

La estrategia eso es todo, introduce la definición de la regla a menos que de la que hablaba Hart, la que permite acomodar el argumento de la superación: "Eso es todo: no hay más reglas y hechos morales relevantes, es decir, no hay una regla moral verdadera y un conjunto de oraciones verdaderas que reemplacen a las que aparecen en este argumento". Con ello se busca es decir que "si eso es así, la regla moral se cumple" (Holton, 2002, p. 375), aunque aparezcan nuevas circunstancias o principios relevantes, ya que simplemente ellos superarían a los principios y hechos relevantes tenidos en cuenta inicialmente. Así, el argumento de los particularistas sería que:

"[P]ara cada principio y conjunto de hechos verdaderos, existe algún otro principio verdadero y un conjunto de hechos posibles que lo superarían [...] Pero ello no implica que cada principio verdadero y conjunto de hechos sea realmente superado; es decir, no implica que exista un verdadero principio y un conjunto de oraciones verdaderas no morales que lo sustituyan" (Holton, 2002, p. 199).

Mediante esta cláusula, se preservarían las dos cuestiones importantes que nos interesa preservar, a saber, que "[n]ecesitamos una noción de reglas jurídicas «bajo la cual sea posible hacer excepciones a las reglas sin rechazar la regla misma»; pero tal noción debería permitir que las reglas aparecieran en justificaciones deductivas de conclusiones del derecho" (Duarte D’Almeida, 2015b, p. 136).

Si esta estrategia acierta, entonces no hay forma de distinguir entre excepciones y suspensiones o cancelaciones entre derechos, ya que la tesis de Schmitt seguiría en pie: no podríamos distinguir entre las excepciones explícitas e implícitas, ni tampoco a ambas con las suspensiones o cancelaciones, ya que en todos los casos siempre cabría considerar situaciones futuras que, mediante la cláusula eso es todo, superarían las normas que establecen derechos. Lo anormal, lo fuera de lo común o lo insubsumible sería el rasgo característico de cualquier norma, incluyendo a las que establecen derechos.

Los derechos quedarían en pie, pero habría circunstancias indeterminadas o no previstas que justamente permitirían exceptuar y cancelar o suspender su protección, sin afectar a los derechos. Esto trastoca toda la concepción de los instrumentos que establecen derechos, ya que ¿de qué serviría mantener un derecho

\footnotetext{
${ }^{16}$ Dancy luego abandona esta caracterización del problema, pasando al tercer particularismo: el que abandona toda idea de principios en la moral.

17 En este mismo sentido se pronuncia Holton, cuando sostiene que "[n]o existe un conjunto finito de principios finitos que sirva para axiomatizar la evaluación ética: esto es, ningún conjunto finito de principios finitos, de modo que, dada cualquier acción descrita completamente en términos no morales, los principios y la descripción conlleven un veredicto moral dado si y solo si es verdad" (2002: 193).
} 
si su protección se podría ver coartada de forma imprevista? ${ }^{18}$ En la sección siguiente intentaré dar una respuesta a este problema.

\section{Excepciones y suspensiones a derechos}

La estrategia para enfrentar el problema antedicho supone dos pasos. El primero, que simplemente mencionaré aquí, por motivos de espacio y énfasis, consiste en afirmar que la supuesta contradicción de (N1) 'Cualquiera que mate a otro ser humano es culpable de homicidio' y (N2) 'Cualquiera que mate a otro ser humano en defensa propia no es culpable de homicidio' no es tal, no al menos en el lenguaje jurídico ordinario.

La clave está en la operación lógica de equivalencia que permite transformar $\mathrm{N} 1$ en N2 cuando a N1 se le agrega una condición. Esto permite, según las modalidades deónticas, que normas con distinto carácter deóntico puedan ser equivalentes cuando a ellas se les agrega una condición.

Esta es una vieja tesis de Bentham, que Raz ha presentado de manera impecable y que en otra parte denomino la tesis de la equivalencia entre excepciones y limitaciones: la idea de que "una limitación es, entonces, una excepción indirecta; una excepción, una limitación indirecta" (Bentham, 2010, p. 124) ${ }^{19}$. Según dicha tesis, una cláusula de excepción de una regla sería donde "se especifica las circunstancias en las que no se aplica, que son la excepción a la regla, lo que implica lógicamente que se aplica en todas las demás circunstancias" (Raz, 1980, p. 57); y la limitación sería donde se "especifica las circunstancias en las cuales se aplica la norma" (Raz, 1980, p. 57).

Por esta razón, una norma prohibitiva con una cláusula de limitación de una circunstancia es equivalente a una prohibición con una cláusula de excepción de la negación de esa circunstancia; y una cláusula prohibitiva con una limitación de una circunstancia es equivalente a una cláusula permisiva con una excepción de esa misma circunstancia. Son estas propiedades lógicas las que permiten afirmar que N1, que es una norma prohibitiva, con la condición "en defensa propia" sea equivalente a N2, que es una permisión.

Pero si definimos de manera distinta a las excepciones, no en base a ciertos marcadores semánticos que permitan reconocer tal o cual operador deóntico -por ejemplo, las condiciones negativas en la norma-, entonces no hay razón para seguir aferrados a la tesis de la equivalencia. Si esto es cierto, podemos pensar simplemente que N1 y N2 no son la misma norma y, por tanto, no son contradictorias.

El segundo consiste en acudir a una intuición básica: no todas las normas que establecen derechos son iguales. Por ello, si renunciamos a un enfoque estructural o lógico para tratar de identificar las excepciones, deberíamos poder tratar de identificarlas funcionalmente, es decir, en los términos en que ellas son distintas a otras normas que establecen restricciones a derechos.

\footnotetext{
18 Quien mejor ha capturado este punto es Webber, quien identifica a éste como uno de los problemas centrales del denominado enfoque recibido de los derechos: cómo justificar el gran volumen de interferencias que los sistemas de protección de derechos permiten (véase Webber, 2009). Si bien Webber se refiere a las limitaciones, su argumento es aplicable a todas las normas alterativas, ya que todas ellas pueden ser vistas como herramientas que autorizan "muchas acciones justificadas de otros que infringen de manera regular y justificada los derechos" (WEBBER, 2016: 15). Porque si este enfoque es plausible, tendría que serlo también la descripción que realiza de la práctica, la que, bajo su prisma, aparece como una práctica donde regularmente se infringen los derechos.

19 Una reconstrucción formalizada del argumento de Bentham se encuentra en Raz, 1980, pp. 55-57 y también Carpentier, 2014, pp. 88-90.
} 
Lo anterior supone comprender que las excepciones, las cancelaciones o suspensiones y otras 'normas alterativas' de derechos pertenecen a una categoría de normas peculiares, cuya funcionalidad es accesoria, alterando justamente los efectos normales, los poderes deónticos de las normas que establecen derechos ${ }^{20}$. En este sentido, las normas alterativas son una expresión de la flexibilidad normativa, esto es, de la capacidad de un sistema jurídico de proporcionar soluciones normativas diferenciadas, moduladas por razones contextuales, frente a los mismos casos.

Creo que una sistematización básica de estas normas alterativas es una forma más adecuada de aproximarse a este problema cuando se trata de normas que establecen derechos. La sensibilidad al contexto de aplicación, con el fin de alcanzar un cierto grado de flexibilidad en la determinación del alcance y protección de los derechos, es su característica más relevante.

En este sentido, los instrumentos que consagran derechos establecen una gran variedad de normas que autorizan al Estado a interferir legítimamente con los derechos sin violarlos. Por ello se ha dicho respecto a los derechos que la situación es así: "lo que se da con una mano se quita con la otra" (Greer, 1997, p. 42). Esto es, lo que se da con una mano, consagrar ciertos derechos, se quita con la otra, permitir restricciones, limitaciones o excepciones a derechos.

Así, hoy prácticamente todos los sistemas de protección de derechos contemplan un sistema de restricciones, lo que ocurre tanto a nivel doméstico como a nivel supranacional. Existe un peligro real de que circunstancias indeterminadas o no previstas permitan exceptuar y cancelar o suspender su protección. Solo basta recordar el infame artículo 48 de la Constitución de Weimar, al que justamente los juristas del Reich echaron mano a fin de justificar el régimen de la Alemania nazi ${ }^{21}$.

Por ello, después de la Segunda Guerra Mundial existía una preocupación en los redactores de los instrumentos que consagraron derechos y en los juristas que les asesoraron, que trataron siempre de constreñir, precisar o especificar de la manera más clara posible la posibilidad de restringir derechos por parte del Estado ${ }^{22}$.

\footnotetext{
20 Existen otras etiquetas que se han propuesto para englobarlas, como "cláusula de acomodación", "sistema de restricciones" o "cláusulas de escape". Sin embargo, prefiero usar la etiqueta "normas alterativas", que me parece más precisa, porque revela claramente de lo que se trata: de la relación que existe entre normas, unas que establecen ciertas soluciones normativas y otras que las alteran. Quien ha tratado en extenso el tema de las limitaciones es Alexy, quien las distingue de las restricciones. Véase Alexy, 1993, pp. 268-269.

${ }^{21}$ La Constitución de Weimar contemplaba en su Artículo 48 lo siguiente: "Si la seguridad y el orden público al interior del Reich son severamente dañados o están en peligro, el presidente del Reich podrá tomar las medidas necesarias que lleven a restablecer el orden, interviniendo con la asistencia de las fuerzas armadas, de ser necesario. Para este propósito, podrá suspenderse temporalmente, totalmente o en parte, los derechos fundamentales proveídos en los artículos 114, 115, 117, 118, 123, 124 y 153". Valga como ejemplo, lo que contemplaba el artículo 114 de la Constitución de Weimar: "Los derechos del individuo son inviolables. La limitación o privación de la libertad individual es admisible solo si se basa en las leyes.

Las personas privadas de libertad tienen que ser notificadas, al día siguiente y con la autoridad más reciente, sobre la base de las razones por las cuales se ordenó la privación de libertad; inmediatamente se les debe dar la oportunidad de protestar contra la privación de libertad".

22 Por ejemplo, en el caso del CEDH, primero se adoptó una cláusula de limitación general por la Asamblea Consultiva, con fecha 30 de agosto de 1949 (véase, Consejo de Europa, 1975, p. 178); luego en la sesión del Comité de Expertos de fecha 6 de marzo de 1950, se decidió cambiar en la forma actual, pero con otro articulado (véase Consejo de Europa, 1976, pp. 280-294). Sobre esto se ha dicho que "[l]a principal razón para oponerse a la cláusula de derogación fue que algunas delegaciones consideraron que una cláusula general de limitación, o varias cláusulas de limitaciones en artículos específicos, podrían hacer el trabajo de la cláusula de derogación" (Oraá, 1992, p. 9).
} 
En el mismo sentido, el Convenio Europeo de Derecho Humanos (CEDH) contempla una norma semejante en su artículo 15, bajo la expresión 'Derogación en caso de estado de excepción'23, cuando en su número 1 señala:

"En caso de guerra o de otro peligro público que amenace la vida de la nación, cualquier Alta Parte Contratante podrá tomar medidas que deroguen las obligaciones previstas en el presente Convenio en la estricta medida en que lo exija la situación, y a condición de que tales medidas no estén en contradicción con las restantes obligaciones que dimanan del derecho internacional" 24 .

\section{Una definición más rigurosa de derogación en este contexto sería:}

"El acto de un Estado que suspende la aplicación y disfrute de ciertos derechos humanos al declarar un estado de emergencia pública que afecta la vida de toda una nación. La derogación permite al Estado adoptar medidas para hacer frente a la emergencia sin temor a violar las normas de derechos humanos durante el período de excepción (Condé, 2004, p. 64) ${ }^{25}$.

Así, lo relevante de la cancelación o la suspensión es justamente constreñir, precisar o especificar la posibilidad de restringir derechos, es decir, contemplar prima facie los poderes normativos fuera de ellas.

Por el contrario, la categoría excepciones es menos clara, en la medida de que ellas se confunden con otras categorías de normas. Así, en la doctrina constitucional se denomina delimitaciones a "situaciones que no se encuentran incluidas en el supuesto de hecho de la norma reguladora de un derecho" (Peces-Barca, 1995, p. 589). Esto con el fin de diferenciarlas de las limitaciones a derechos propiamente dichas.

Otro tanto ocurre con el sistema europeo de derechos humanos, donde es posible distinguir entre dichas delimitaciones y las excepciones propiamente dichas, aunque conviene realizar presiones previas, ya que estas últimas no serían, como se señaló en la sección anterior, meros elementos negativos de las normas.

${ }^{23}$ En la versión inglesa oficial del CEDH, el encabezado del artículo 15 es "Derogation in time of emergency"; del mismo modo, la versión oficial en francés, el artículo 15 lleva por encabezado "Dérogation en cas d'état d'urgence". Por su parte, la traducción castellana oficial del texto del Convenio, disponible en el sitio web del Tribunal, titula al artículo 15 como "Derogación en caso de estado de excepción”. Así que, al menos en el contexto del CEDH, se debe preferir el uso de 'derogación' para referirse al mecanismo de suspensión de derechos en caso de estado de excepción, que se encuentra contenido en el artículo. Pero, en el contexto de este trabajo, he preferido referirme a dicho mecanismo como suspensión o cancelación, por ser para la teoría del derecho el término más adecuado. Véase Moreso y Vilajosana, 2004, p. 120.

${ }^{24}$ El número 3 establece la obligación información, justificación y temporalidad de la adopción del régimen de derogación bajo el sistema del CEDH. El número 2 señala los derechos inderogables: el derecho a la vida (recordemos que se debe considerar lo señalado en el Protocolo No. 6, que abolió la pena de muerte en tiempo de paz, y el Protocolo No. 13, que lo hizo en toda circunstancia, prohibiendo en su artículo 2 cualquier derogación de esta prohibición); la prohibición de la tortura, la prohibición de la esclavitud o servidumbre, y la prohibición de condena sin delito o de pena más gravosa. A ello debe sumársele lo señalado en el Protocolo No. 7, que en su artículo 4 señala que no es derogable el derecho a no ser juzgado o condenado dos veces.

${ }^{25}$ Este sería el caso, mutatis mutandis, de los derechos no-absolutos, de lo que Feinberg denominaba "renuncia temporal de derechos". En el ejemplo, tomado de Don E. Scheid, del rito veraniego tribal, donde "Durante una hora cada hombre es cazador y presa. Durante ese período de tiempo el derecho normal a la vida se suspende para todos los participantes voluntarios. De hecho, cada uno de ellos ha renunciado a la protección de ese derecho durante un período de tiempo determinado, sin posibilidad de recuperarlo hasta que haya transcurrido el tiempo y el juego haya terminado. Cada jugador libera así a todos los demás jugadores de su obligación normal de no matarlo" (Feinberg, 1978, p. 117). 
Así, en el caso del CEDH, el denominado 'sistema de restricciones' contempla diversas categorías de normas que pueden afectar a los derechos. En general, se reconocen los siguientes tipos de restricciones: las generales, v.gr. los artículos 15 y 17-18 del Convenio que establecen cualificaciones generales sobre los derechos; los casos de derogación en estado de excepción, la prohibición del abuso de derecho y la aplicación de restricciones solo para los fines previstos, respectivamente; las cláusulas de limitación, v.gr. los artículo 8-11 del Convenio, artículos 2 del Protocolo No. 4 y 1 del Protocolo 7, y las limitaciones por delimitación, esto es, las que "delimitan el alcance de la protección de ciertos derechos y explícitamente se excluyen áreas específicas o personas del foco de la protección, o se 'delega' a las autoridades del Estado la responsabilidad de regular el ejercicio de ciertos derechos", por ejemplo, los artículos 2(2), 4(3) o 12) (Van Dijk et. al., 2006, pp. 333 y 342$)^{26}$.

Las cancelaciones o suspensiones (derogaciones) se encontrarían en las llamadas restricciones generales, mientras que las excepciones entre las llamadas delimitaciones. Veamos entonces algunos ejemplos en el sistema del CEDH.

El artículo 4 establece la prohibición de la esclavitud y del trabajo forzado. Se dice que solo el primer derecho no está cualificado, ni posee excepciones de ningún tipo ni se le aplica la derogación (cancelación o suspensión) del artículo 15. En cambio, el segundo derecho, la prohibición del trabajo forzado, sí las contempla, además de permitir su derogación (cancelación o suspensión bajo el artículo 15). En su número 3 contempla los casos que no se consideran trabajo forzado u obligatorio: a) el exigido normalmente a una persona privada de libertad; b) el servicio de carácter militar; c) el servicio exigido cuando alguna emergencia o calamidad amenacen la vida o el bienestar de la comunidad; d) el trabajo o servicio que forme parte de las obligaciones cívicas normales (por ejemplo, vocal de mesa en elecciones) ${ }^{27}$.

La práctica jurídica reconoce inequívocamente a los derechos establecidos en los artículos 8-11 como derechos que poseen cláusulas de limitación. Por ejemplo, el artículo 9(1) del CEDH contempla que "[t]oda persona tiene derecho a la libertad de pensamiento, de conciencia y de religión", lo que supone la "[l]a libertad de manifestar su religión o sus convicciones". El artículo 9(2) contempla la siguiente cláusula de limitación: "La libertad de manifestar su religión o sus convicciones no puede ser objeto de más restricciones que las que, previstas por la ley, constituyan medidas necesarias, en una sociedad democrática, para la seguridad pública, la protección del

\footnotetext{
26 También se ha desarrollado una doctrina de las limitaciones inherentes, es decir, "restricciones a ciertos derechos del Convenio que no se consideran equivalentes a interferencias, debido a que ellas están arraigadas en el ámbito de la garantía de esos derechos". No tienen un alcance general y se aplican sobre derechos específicos, por ejemplo, artículo 6(1) respecto del acceso al Tribunal y el artículo 3 del Protocolo No. 1 respecto del derecho a votar y a presentarse a una elección, que surgió desde la Comisión y que hoy cuenta con un respaldo minoritario, además, su aplicación queda excluida en el caso de los derechos que cuentan con cláusulas de limitación expresa, "sobre la base de que la posibilidad de restricciones legítimas sobre los derechos del Convenio están enumeradas de manera exhaustiva" (Van Dijk et al., 2006, pp. 343 y 345).

${ }^{27}$ En el mismo sentido, el artículo $8 n^{\circ} 3$ PIDCP: "a) Nadie será constreñido a ejecutar un trabajo forzoso u obligatorio; c) No se considerarán como 'trabajo forzoso u obligatorio', a los efectos de este párrafo: i) Los trabajos o servicios que, aparte de los mencionados en el inciso b), se exijan normalmente de una persona presa en virtud de una decisión judicial legalmente dictada, o de una persona que habiendo sido presa en virtud de tal decisión se encuentre en libertad condicional; ii) El servicio de carácter militar y, en los países donde se admite la exención por razones de conciencia, el servicio nacional que deben prestar conforme a la ley quienes se opongan al servicio militar por razones de conciencia; iii) El servicio impuesto en casos de peligro o calamidad que amenace la vida o el bienestar de la comunidad; iv) El trabajo o servicio que forme parte de las obligaciones cívicas normales". Igualmente, el artículo 18 de la Constitución Española, que en su número 2 establece que "El domicilio es inviolable. Ninguna entrada o registro podrá hacerse en él" para, a continuación, establecer las excepciones: "sin consentimiento del titular o resolución judicial, y la contra excepción "salvo en caso de flagrante delito".
} 
orden, de la salud o de la moral públicas, o la protección de los derechos o las libertades de los demás"28.

La clave está aquí en el tipo de norma que establece el derecho. Una de las diferencias es la formulación que adopta en el caso de uno y otro derecho. Mientras que, en el caso de los trabajos forzados, el derecho se establece en términos de una prohibición, es decir, el CEDH establece precisamente qué acciones están prohibidas, en el caso de la libertad de manifestar su religión o convicciones se identifican cosas abstractas a las que todos tienen derecho ${ }^{29}$.

Estas diferencias de formulación son también relevantes, en el sentido de que las cláusulas de limitación y excepción guardan relación con la norma principal, la norma que establece el derecho, a la que se adscriben. Este criterio ayuda a comprender, en el caso de los derechos, cuándo nos encontramos frente a una excepción y cuándo frente a una limitación.

En el caso de los derechos establecidos en términos de prohibiciones, el legislador utiliza excepciones, a fin de que, con precisión, quede claro qué casos le interesa prima facie excluir en vez de incluir en la prohibición. Su adscripción a ciertos derechos indica que el legislador, específicamente, ha decidido dejar fuera ciertos casos que de otro modo caerían dentro de la prohibición que el derecho expresa. Este es el caso del artículo 4 del CEDH que establece los casos que no se considerarán trabajo forzado. Esta exclusión prima facie es relevante y debe ser tenida en cuenta por el intérprete, pero es relevante no por sí misma, sino por el tipo de norma que establece derechos a la que se aplica ${ }^{30}$. Además, ello satisface el conocido requisito de la especificidad de las excepciones (véase Carpertier, 2014, p. 29).

${ }^{28}$ Dicho derecho, que también se contempla en el caso del artículo 18 del Pacto Internacional de Derechos Civiles y Políticos, contiene en su número 3 la cláusula limitativa: "La libertad de manifestar la propia religión o las propias creencias estará sujeta únicamente a las limitaciones prescritas por la ley que sean necesarias para proteger la seguridad, el orden, la salud o la moral públicos, o los derechos y libertades fundamentales de los demás".

29 Sobre esta distinción se ha dicho recientemente: "Al examinar los treinta artículos de la Declaración Universal de Derechos Humanos, se observan dos conjuntos diferentes de formulaciones empleadas por los redactores: 'Todo el mundo tiene derecho a...' y 'Nadie debe ser...'. La primera formulación identifica algunas cosas abstractas a las que todos tienen derecho: libertad, vida, igualdad, libertad de religión, libertad de asociación, etc. La segunda formulación difiere por la identificación de alguna acción que está prohibida: una orden judicial contra un acto, una obligación de no realizar un acto determinado. La diferencia es significativa [...] Las mismas dos formulaciones son empleadas por el Convenio, con los mandamientos negativos desplegados con respecto a privar intencionalmente a otro de la vida, practicar la tortura, los tratos o penas inhumanas o degradantes; mantener a otro en la esclavitud o la servidumbre, someter a otros a trabajo forzoso u obligatorio, y encontrar culpable a otro de un delito retroactivo o imponer una pena penal retroactiva" (Webber, 2016, p. 2).

${ }^{30}$ Así lo ha dicho el TEDH recientemente en el caso Magyar Helsinki Bizottság v. Hungary, aplicación No. 18030/11, sentencia de 8 noviembre de 2016, donde el Tribunal ha clarificado el procedimiento general de interpretación del CEDH, estableciendo una especie de orden de prelación en el uso de las técnicas de interpretación, destacando primerísimamente la necesidad de seguir las reglas de interpretación de la Convención de Viena sobre los Tratados (artículos 31 a 33) y, por ende, la principal técnica de interpretación, la literal, como señala en el §463: "Por lo tanto, de conformidad con la Convención de Viena, la Corte está obligada a determinar el significa dar a las palabras en su contexto y a la luz del objeto y propósito de la disposición de la que se extraen". Además se recalca la preeminencia del argumento sedes materiae, esto es, "cuando una determinada formulación normativa se interpreta atendiendo al lugar que ocupa en el contexto del que forma parte" (Moreso y Vilajosana, 2004, p. 169). 
Por su parte, en el caso de los derechos establecidos en términos de derechos a cosas abstractas, el legislador utiliza limitaciones, ya que prima facie está reconociendo que incluye en la determinación de la protección de esos derechos conceptos morales densos, tales como salud, orden o moral pública ${ }^{31}$. Nuevamente, esta inclusión prima facie de dichos conceptos en la norma es relevante y debe ser tenida en cuenta por el intérprete al momento de su aplicación ${ }^{32}$.

Para desvincular la intención del legislador de la identificación de las excepciones y limitaciones, es preciso mostrar que lo relevante no es solo la intención del legislador de adoptar los derechos de tal manera, sino que sistemáticamente fueron establecidos de esa manera y que dicha sistematicidad se ve reflejada en la práctica jurídica.

El enfoque funcional renuncia a identificar a las normas alterativas en base a criterios estructurales. Trata de solucionar el problema del tránsito desde la identificación de cláusulas exceptivas o las cláusulas suspensivas hacia los efectos que ellas producen en las normas, las excepciones y suspensiones o cancelaciones a derechos. Ello, mediante el establecimiento de un criterio funcional: las normas alterativas afectarían diferentes aspectos de los derechos. Esta sería la característica común de todas ellas.

¿Cuáles serían estas normas alterativas en el caso de las normas que establecen derechos? Tomemos como ejemplo el sistema del CEDH. Según la dogmática, los elementos que alteran los derechos y que están contemplados en determinadas normas del CEDH serían:

\footnotetext{
${ }^{31}$ Como señala Williams: "La forma en que se aplican estas nociones está determinada por cómo es el mundo (por ejemplo, por cómo se ha comportado alguien) y, sin embargo, al mismo tiempo, su aplicación generalmente implica una cierta valoración de la situación, de personas o acciones" (Williams, 2006, p. 129). Sobre los conceptos densos y su importancia para la interpretación constitucional, véase Moreso, 2000, pp. 109-110.

32 Pudiera pensarse en contraejemplos, como en la Constitución Sudafricana, donde su artículo 16 define la libertad de expresión en su número 1, a la vez que en el número 2 establece la siguiente norma: "El derecho en la subsección (1) no se extiende a (a) propaganda para la guerra; (b) incitación a la violencia inminente; o (c) la defensa del odio que se basa en la raza, la etnia, el sexo o la religión, y que constituye una incitación a causar daño". ¿Cómo es posible que se adscriba funcionalmente una excepción, que prima facie excluye elemento de la norma, respecto de un derecho a algo abstracto como la libertad de expresión? La respuesta es que la Constitución Sudafricana adopta una cláusula de limitación general, donde sí se establecen los conceptos densos, a los que aludí antes, y que el juez debe tener en cuenta. Por lo tanto, la relación funcional entre cláusulas de limitación y excepción funciona de manera diferente, lo que no afecta mi argumento, ya que es plausible que a una norma se le adscriban ambos tipos de cláusulas, dado que ellas alterarían diferentes partes de la norma: la excepción el alcance y la limitación la extensión de la protección.
} 


\begin{tabular}{|c|c|c|c|c|c|}
\hline & & RESERVAS & DEROGACIONES & EXCEPCIONES & LIMITACIONES \\
\hline \multirow{2}{*}{ ALCANCE } & \begin{tabular}{|cr} 
TODOS & LOS \\
DERECHOS (menos \\
no-derogables)
\end{tabular} & $\mathrm{x}$ & $\checkmark$ & $\mathrm{x}$ & $\mathrm{x}$ \\
\hline & \begin{tabular}{|c|} 
DERECHOS \\
ESPECIFICOS \\
\end{tabular} & $\checkmark$ & $\mathrm{x}$ & $\checkmark$ & $\checkmark$ \\
\hline \multirow{2}{*}{$\begin{array}{l}\text { TIPO DE } \\
\text { CIRCUNSTANCIAS }\end{array}$} & EXCEPCIONALES & $\mathrm{x}$ & $\checkmark$ & $\mathrm{x}$ & $\mathrm{x}$ \\
\hline & NORMALES & $\checkmark$ & $\mathrm{x}$ & $\checkmark$ & $\checkmark$ \\
\hline \multirow{2}{*}{ TEMPORALIDAD } & PERMANENTES & $\checkmark$ & $\mathrm{x}$ & $\checkmark$ & $\checkmark$ \\
\hline & TRANSITORIAS & $\mathrm{x}$ & $\checkmark$ & $\mathrm{x}$ & $\mathrm{x}$ \\
\hline \multirow{2}{*}{$\begin{array}{c}\text { INTENSIDAD DEL } \\
\text { CONTROLL }\end{array}$} & $\left|\begin{array}{cc}\text { C(más } & \text { intenso) }\end{array}\right|$ & $\checkmark$ & $\mathrm{x}$ & $\checkmark$ & $\mathrm{x}$ \\
\hline & $\begin{array}{|rr|}\text { COMPA TIBILIDAD } \\
\text { (menos } & \text { intenso) }\end{array}$ & $\mathrm{x}$ & $\checkmark$ & $\mathrm{x}$ & $\checkmark$ \\
\hline \multirow{2}{*}{$\begin{array}{l}\text { DECLARACIÓN } \\
\text { ESPECIAL }\end{array}$} & $\begin{array}{c}\text { REQUIERE } \\
\text { NOTIFICACIÓN }\end{array}$ & $\checkmark$ & $\checkmark$ & $\mathrm{x}$ & $\mathrm{x}$ \\
\hline & $\begin{array}{|cr|}\text { NO } & \text { REQUIERE } \\
\text { NOTIFICACIÓN }\end{array}$ & $\mathrm{x}$ & $\mathrm{x}$ & $\checkmark$ & $\checkmark$ \\
\hline
\end{tabular}

En este sentido, se puede construir una definición tentativa de cada una de las normas alterativas que componen este sistema:

(1) Reservas. Una reserva se establece prima facie y puede afectar a priori tanto el alcance como la extensión de la protección de un derecho, de manera específica y permanente, sustrayendo o agregando algún elemento de la norma que lo establece y solo con efecto parcial para el Estado que realiza la reserva.

(2) Cancelaciones o suspensiones (derogaciones). Ella afecta a posteriori la protección de un derecho, de manera esencialmente transitoria, introduciendo unos elementos externos genéricos que el instrumento que establece derechos prima facie preselecciona y cuya calificación corresponde al tribunal.

(3) Excepciones. La excepción está establecida prima facie en la adopción de la norma que establece un derecho y sustrae a priori del alcance o de la extensión de la protección de un derecho, de manera permanente y con efectos generales para todos, un determinado ítem que de otra manera debería entenderse incluido en ella.

(4) Limitaciones. Una limitación agrega o introduce, a posteriori, a los elementos que configuran la extensión de la protección de un derecho, un determinado ítem, mediante una cláusula que indica en la norma una preselección prima facie de elementos constituidos por conceptos densos, que el Tribunal debe tener en cuenta al momento de calificar la limitación del derecho.

\section{Conclusión}

La reconstrucción sistemática de las normas alterativas permite, por una parte, señalar las semejanzas funcionales que existen entre las distintas normas que las componen, y por otra, mostrar de qué manera se produce la alteración que ellas comportan en los poderes normativos de las normas que establecen derechos.

Así, en el caso que nos ocupa aquí, y dejando de lado las reservas, cuya naturaleza, ya se dijo, es esencialmente apriorística, las limitaciones se distinguen de las cancelaciones o suspensiones (derogación), porque las cancelaciones son casos de suspensión de garantías por un tiempo acotado, transitoriedad que no poseen las limitaciones.

Por su parte, las limitaciones comparten con las cancelaciones una característica central, a saber, que ambas introducen una preselección prima facie de elementos que modulan la extensión de la protección del derecho, elementos que los tribunales deben calificar en casos concretos, esto es, tras la adopción de las normas contempladas en el instrumento de que se trate. 
En el caso de las cancelaciones o suspensiones, dicha introducción prima facie de elementos preseleccionados se hace de manera específica, tal y como lo haría una excepción en la norma, pero con efectos temporales en la protección del derecho. En cambio, en el caso de las limitaciones, ella también se hace de manera específica, pero con efectos permanentes en la protección del derecho. En ambos casos, sin embargo, lo que se limita o cancela es la extensión de la protección de un derecho, lo que es especialmente relevante en el caso de normas que establecen derechos a cosas abstractas, cuya adopción incluye conceptos morales densos.

Finalmente, una excepción, entendida de la manera aquí propuesta, se establece prima facie en la norma y sustrae a priori del alcance o de la extensión de la protección de un derecho, de manera permanente y con efectos generales, un determinado ítem que de otra manera debería entenderse incluido. Por ello, lo que ella afecta es a priori el alcance de un derecho, lo que es especialmente relevante en el caso de las normas que establecen derechos adoptadas en forma de prohibiciones, las que buscan guiar de forma más directa el comportamiento de los destinatarios.

Esta semejanza funcional es la que ha llevado a muchos autores, dedicados a estudiar el sistema del CEDH, a confundir los distintos tipos de normas alterativas y a alentar una lectura superficial del CEDH en términos de que todas las restricciones serían una clase de excepciones y, por tanto, formas en que los Estados pueden incumplir o estar relevados de cumplir con sus obligaciones ${ }^{33}$.

Solo en el contexto sistemático específico del tipo de norma de que se trate tiene sentido mantener la distinción entre excepciones, suspensiones 0 cancelaciones, y demás normas alterativas. Ellas resisten su conceptuación en abstracto, fuera de cualquier sistema de normas específico: hacerlo induce a equívocos, ya que se las confunde con situaciones anormales que desencadenan potenciales arbitrariedades; se alienta una lectura superficial de los instrumentos que establecen derechos, una que carece de un adecuado sentido de la realidad jurídica; $\mathrm{y}$, finalmente, se desdibuja su importancia, ya que se desconoce su carácter como valiosas herramientas interpretativas que permiten obtener la suficiente flexibilidad que demanda el desarrollo de cualquier sistema normativo.

\section{Bibliografía}

ADRADOS, F. (1990), Líricos griegos. Elegiacos y yambógrafos arcaicos (siglo VII-V A.C.), Vol I (trad. F. Adrados), Consejo Superior de Investigaciones Científicas, Madrid.

ALCHURRÓN, C.E. (1991), "Condicionalidad y la representación de las normas jurídicas". En: BULYGIN, E. y ALCHURRÓN, C.E., Análisis lógico y derecho, Madrid, Centro de Estudios Constitucionales, pp. 267-280.

ALCHURRÓN, C.E. (2000), "Sobre derecho y lógica", Isonomía, No. 3, pp. 11-33.

ALEXY, R. (1993), Teoría de los derechos fundamentales (trad. E. Garzón Valdés), Centro de Estudios Constitucionales, Madrid.

ANSUÁTEGUI, F.J. (1999), "El derecho como norma". En: PECES-BARBA, G., FERNÁNDEZ, E. y DE ASÍS, R., Curso de teoría del derecho, Madrid, Marcial Pons, pp.147-173.

ATRIA, F. (2001), On Law and Legal Reasoning, Hart Publishing, Oxford and Portland. BENTHAM, J. (2010), Of the Limits of the Penal Branch of Jurisprudence (ed P.

\footnotetext{
33 Véase, por ejemplo, Borowski, 2000 y 2007; Grote, 2003; Higgins, 1978 y 1997; Van Dijk et al., 2006.
} 
Schofield), Clarendon Press, Oxford.

BOROWSKI, M. (2000), "La restricción de los derechos fundamentales", Revista Española de Derecho Constitucional, Año 20, No. 59, pp. 29-56.

BOROWSKI, M. (2007), "Limiting Clauses: On the Continental European Tradition of Special Limiting Clauses and the General Limiting Clause of Art 52(1) Charter of Fundamental Rights of The European Union", Legisprudence, Vol. 1, No. 2, pp. 197-240.

CARPENTIER, M. (2014), Norme et exception. Essai sur la défaisibilité en droit, Institut Universitaire Varenne, Paris.

CONDÉ, H. V. (2004), A Handbook of International Human Rights Terminology, University of Nebraska Press, Lincoln and London.

CONSEJO DE EUROPA (1975), Collected Edition of the "Travaux Préparatoires" / Recueil des "Travaux Préparatoires", Volume I, Martinus Nijhoff, La Haya.

CONSEJO DE EUROPA (1976), Collected Edition of the "Travaux Préparatoires" of the European Convention on Human Rights / Recueil des Travaux Préparatoires de la Convention Européenne des Droits de l'Homme, Volume III, Martinus Nijhoff, La Haya.

DANCY, J. (2015), “¿Qué es el particularismo en ética?”. En: LUQUE, P. (ed.), Particularismo, Madrid, Barcelona, Buenos Aires y Sâo Paulo, Marcial Pons, pp. 19-37.

DIEZ-PICAZO, L.M. (2003), Sistema de derechos fundamentales, Thomson Civitas, Madrid.

DUARTE D'ALMEIDA, L. (2015a), Allowing for Exceptions: A Theory of Defences and Defeasibility in Law, Oxford University Press, Oxford.

DUARTE D'ALMEIDA, L. (2015b), “Excepciones y superación”. En: LUQUE, Pau (ed.), Particularismo, Madrid; Barcelona, Buenos Aires y Sâo Paulo: Marcial Pons, pp. 133-150.

DWORKIN, R. (2012), Los derechos en serio (trad. M. Gustavino), Ariel, Barcelona.

FEINBERG, J. (1978), "Voluntary Euthanasia and the Inalienable Right to Life", Philosophy \& Public Affairs, Vol. 7, No. 2, pp. 93-123.

FINKELSTEIN, C. (2000), "When the Rule Swallows the Exception", Quinnipiac Law Review, Vol. 19, No. 3, pp. 505-537.

GÓMEZ, G. (1986), Excepción y normalidad en el pensamiento de Carl Schmitt, Centro de Estudios Constitucionales, Madrid.

GREER, S, (1997), The exceptions to Articles 8 to 11 of the European Convention on Human Rights, Council of Europe Publishing Human Rights. Files No. 15, Strasbourg.

GROTE, R. (2003), "Limitaciones para la ley en la regulación de los derechos humanos en el derecho europeo, con especial referencia al derecho alemán", Anuario de derecho constitucional latinoamericano, No. 2003, pp. 83-108.

HART, H.L.A. (1948-1949), "The Ascription of Responsibility and Rights", Proceedings of the Aristotelian Society, New Series, Vol. 49, pp. 171-194.

HART, H.L.A. (1997), The Concept of Law (2nd edition), Oxford University Press, Oxford.

HIGGINS, R. (1997), "Introduction". En: GARDNER, J.P. (ed.), Human Rights as a General Norms and a State's Right to Opt Out. London, The British Institute of International and Comparative Law, pp. xv-xxix.

HIGGINS, R. (1978). "Derogations under Human Rights Treaties". En: JENNINGS R.Y. and BROWNLIE, I. (eds.), The British Year Book of International Law 19761977, Oxford, Clarendon Press, pp. 281-320.

HOLTON, R. (2002), "Principles and particularism", Proceedings of the Aristotelian Society, Supplementary Volume, Vol. 67, No. 1, pp. 191-209.

HOLTON, R. (2010), "The Exception Proves the Rule", The Journal of Political Philosophy, Vol. 18, No. 4, pp. 369-388.

HONIG, B. (2009), Emergency Politics. Paradox, Law, Democracy, Princeton 
University Press, Princeton.

HONORÉ, A.M. (1977), "Real Laws". En: HACKER, P.M.S. and RAZ, J., Law, Morality, and Society, Oxford, Clarendon Press, pp. 99-118.

KELSEN, H. (1986), Teoría pura de derecho (trad. R.J. Vernengo), Universidad Nacional Autónoma de México, México D.F.

LUQUE, P. (2015), "Introducción. El particularismo entre la guerra de los hechos y la paz de las reglas". En: LUQUE, P. (ed.), Particularismo, Madrid, Barcelona, Buenos Aires y Sâo Paulo, Marcial Pons, pp. 11-17.

MACCORMICK, N. (1995), "Defeasibility in Law and Logic". En: BANKOWSKI, Z. et al. (eds.), Informatics and the Foundations of Legal Reasoning, Dordrecht, Kluwer, pp. 99-117.

MORESO, J. (2014), "Bentham's Deontic Logic". En: TUSSEAU, G. (ed.), The Legal Philosophy and Influence of Jeremy Bentham. Essays on Of the Limits of the Penal Branch of Jurisprudence, London, Routledge, pp. 71 -80.

MORESO, J.J. y NAVARRO, P. (1996), "Applicabilità ed Efficacia delle norme giuridiche". En: P. COMANDUCCI, P. y GUASTINI, R. (eds.), Struttura e Dinamica dei sistemi giuridici, Torino, Giappichelli, pp. 15-36.

MORESO, J.J. y VILAJOSANA, J.M. (2004), Introducción a la teoría del derecho, Marcial Pons, Madrid.

ORAÁ, J. (1992), Human Rights in States of Emergency in International Law, Clarendon Press, Oxford.

RAZ, J. (1980), The Concept of a Legal System: An Introduction to the Theory of a Legal System, Clarendon Press, Oxford.

SCHAUER, F. (1991), "Exceptions", Chicago Law Review, Vol. 58, No. 3, pp. 871-899.

SCHMITT, C. (1996), Sobre los tres modos de pensar la ciencia jurídica (trad. M. Herrero), Tecnos, Madrid.

SCHMITT, C. (2009), Teología política (trad. F.J. Conde y J. Navarro), Trotta, Madrid.

VAN DIJK, P. et al. (eds) (2006), Theory and Practice of the European Convention on Human Rights (Fourth Edition), Intersentia, Antwerpen and Oxford.

WEBBER, G. (2009), The Negotiable Constitution. On the Limitation of Rights, Cambridge University Press, Cambridge.

WEBBER, G. (2016), "Proportionality and Absolute Rights". En: JACKSON, V. y TUSHNET, M. (eds.), Proportionality: New Frontiers, New Challenges. Cambridge, Cambridge University Press, pp. 1-24.

WILLIAMS, B. (2006), "Chapter 7. The Linguistic Turn" and "Chapter 8. Knowledge, Science, Convergence". En: WILLIAMS, B., Ethics and the Limits of Philosophy, London, Routledge, pp.120-131 y 132-155. 